\title{
REMARKS ON COMPLEX CONTACT MANIFOLDS
}

\section{SHOSHICHI KOBAYASHI}

1. Statement of results. Let $M$ be a complex manifold of complex dimension $2 n+1$. Let $\left\{U_{i}\right\}$ be an open covering of $M$. We call $M$ a complex contact manifold if the following conditions are satisfied:

(1) On each $U_{i}$ there exists a holomorphic 1 -form $\omega_{i}$ such that $\omega_{i} \wedge\left(d \omega_{i}\right)^{n}$ is different from zero at every point of $U_{i}$.

(2) If $U_{i} \cap U_{j}$ is nonempty, then there exists a nonvanishing holomorphic function $f_{i j}$ on $U_{i} \cap U_{j}$ such that $\omega_{i}=f_{i j} \omega_{j}$ on $U_{i} \cap U_{j}$.

We shall prove the following

THEOREM. If $M$ is a complex contact manifold of complex dimension $2 n+1$, then

(1) The structure group of the tangent bundle of $M$ can be reduced to $U(1) \times(\mathrm{Sp}(n) \otimes U(1))$.

(2) Let $c_{i}(M)$ be the ith Chern class of (the tangent bundle of) $M$ and $\alpha$ the characteristic class of the line bundle over $M$ defined by $\left\{f_{i j}\right\}$. Then

$$
1+c_{1}(M)+c_{2}(M)+\cdots=(1+\alpha)(1+n \alpha+\cdots) .
$$

In particular, $c_{1}(M)$ is divisible by $n+1 ; c_{1}(M)=(n+1) \alpha$.

(3) There exists a principal fibre bundle $P$ over $M$ with structure group $U(1)$ such that $P$ is a real contact manifold. Moreover, both $P$ and a real contact form on $P$ can be constructed in a natural way from $M$ and $\omega_{i}$.

Chern has shown in [2] that the structure group of the tangent bundle of an orientable real contact manifold of real dimension $2 n+1$ can be reduced to $S O(1) \times U(n)(=S O(1) \times(S U(n) \otimes U(1))$. Hence, (1) of our theorem is an analogue of the result of Chern.

At the end of this paper, we shall give two examples of (3).

\section{Proof of (1).}

Let $T_{x}(M)$ be the complex tangent space to $M$ at a point $x$. Assume $x$ to be in $U_{i}$. As $\omega_{i} \neq 0$ at $x, \omega_{i}=0$ defines a $2 n$-dimensional complex vector subspace $F_{x}$ of $T_{x}(M)$. Let $F$ be the vector bundle over $M$ with fibres $F_{x}$. Let $E$ be the line bundle $T(M) / F$. Then, $T(M) \cong F \oplus E$ (Whitney sum). From the definition of contact form, it follows that $d \omega_{i}$, on $F_{x}$, is of maximal rank and is defined up to a factor, i.e.,

$$
d \omega_{j}\left|F_{x}=f_{j i} d \omega_{i}\right| F_{x}
$$

Received by editors May 31, 1958. 
Now, (1) follows immediately from the definition of $\operatorname{Sp}(n)$. (See, for instance, [3].)

3. Proof of (2). From $\omega_{i}=f_{i j} \omega_{j}$, it follows easily that

$$
\omega_{i} \wedge\left(d \omega_{i}\right)^{n}=\left(f_{i j}\right)^{n+1} \omega_{j} \wedge\left(d \omega_{j}\right)^{n} .
$$

Since $\omega_{i} \wedge\left(d \omega_{i}\right)^{n}$ is a holomorphic form of degree $2 n+1,\left\{f_{v}^{-(n+1)}\right\}$ defines the canonical bundle $K$ of $M$. The characteristic class of $K$ is $-c_{1}(M)$, (see for instance $[1, \S 29]$ ). The line bundle $E=T(M) / F$ is defined by $\left\{f_{i j}\right\}$. From $K=E^{-(n+1)}$, we obtain

$$
c_{1}(M)=(n+1) \alpha
$$

Let $c_{i}(F)$ be the $i$ th Chern class of the vector bundle $F$. By the Whitney Duality Theorem,

$$
1+c_{1}(M)+c_{2}(M)+\cdots=(1+\alpha)\left(1+c_{1}(F)+c_{2}(F)+\cdots\right) .
$$

From $c_{1}(M)=(n+1) \alpha$, it follows that $c_{1}(F)=n \alpha$.

4. Proof of (3). Let $L$ be the line bundle over $M$ defined by $\left\{f_{i j}^{-1}\right\}$ and $p$ the projection of $L$ onto $M$. Let $h_{i}: p^{-1}\left(U_{i}\right) \rightarrow U_{i} \times C$ be the coordinate map. If $v \in L_{x}$ and $x \in U_{i} \cap U_{j}$, then

$$
h_{i}(v)=\left(x, z_{i}\right), \quad h_{j}(v)=\left(x, z_{j}\right), \quad z_{i}=f_{j i} z_{j} .
$$

Hence

$$
z_{i} \cdot p^{*}\left(\omega_{i}\right)=z_{j} \cdot p^{*}\left(\omega_{j}\right),
$$

showing that $\left\{z_{i} \cdot p^{*}\left(\omega_{i}\right)\right\}$ defines a holomorphic 1 -form $\omega$ on $L$.

A simple calculation shows that

$$
\begin{aligned}
(\omega+\bar{\omega}) \wedge( & d \omega+d \bar{\omega})^{2 n+1} \\
& =A\left(z_{i} \bar{z}_{i}\right)^{n}\left(\bar{z}_{i} d z_{i}-z_{i} d \bar{z}_{i}\right) \wedge p^{*}\left(\omega_{i} \wedge\left(d \omega_{i}\right)^{n} \wedge \bar{\omega}_{i} \wedge\left(d \bar{\omega}_{i}\right)^{n}\right)
\end{aligned}
$$

where $A=(2 n+1) ! /(n !)^{2}$.

We define a bundle $P$ as follows. Let $B_{x}$ be a positive definite hermitian form on $L_{x}$ differentiable with respect to $x$. Let

$$
P_{x}=\left\{v \in L_{x} ; B_{x}(v, v)=1\right\},
$$

i.e., $P_{x}$ is a circle in $L_{x}$. Then $P=\bigcup_{x \in M} P_{x}$ is a principal fibre bundle over $M$ with group $U(1)$.

We shall show that the restriction of $\omega+\bar{\omega}$ to $P$ defines a real contact structure on $P$. If $P_{x}$ is given by

$$
z_{i} \bar{i}_{i}=b(x)^{2} \quad(b(x)>0),
$$

then

$$
\left(\bar{z}_{i} d z_{i}-z_{i} d \bar{z}_{i}\right)=2 \bar{z}_{i} d z_{i}-2 b \cdot d b
$$


As $\omega_{i} \wedge\left(d \omega_{i}\right)^{n} \wedge \bar{\omega}_{i} \wedge\left(d \bar{\omega}_{i}\right)^{n} \wedge d b$ is a form of degree $4 n+3$ (> real dimension of $M$ ) on $M$, it vanishes identically. Hence, the restriction of $(\omega+\bar{\omega}) \wedge(d \omega+d \bar{\omega})^{2 n+1}$ to $P$ is

$$
2 A b^{2 n} \bar{z}_{i} d z_{i} \wedge p^{*}\left(\omega_{i} \wedge\left(d \omega_{i}\right)^{n} \wedge \bar{\omega}_{i} \wedge\left(d \bar{\omega}_{i}\right)^{n}\right),
$$

which is clearly different from zero at every point of $P$.

Remark. Let $Q_{x}$ be the disk defined by the circle $P_{x}$. Then $P$ is the boundary of $Q=\cup Q_{x}$ and $(\omega+\bar{\omega})$ is of maximal rank on $Q$ except at the points of $M$, as easily verified. This agrees with a result of Gray [4].

\section{Examples.}

(1) Complex projective spaces of odd dimension. Let $z^{1}, z^{2}, \cdots$, $z^{2 n+1}, z^{2 n+2}$ be a coordinate in the $(2 n+2)$-dimensional complex vector space $C^{2 n+2}$ and let $P_{2 n+1}(C)$ be the $(2 n+1)$-dimensional complex projective space. Then, $C^{2 n+2}-\{0\}$ is the principal fibre bundle associated with a line bundle $L$ over $P_{2 n+1}(C)$. Set

$$
\omega=z^{1} d z^{2}-z^{2} d z^{1}+\cdots+z^{2 n+1} d z^{2 n+2}-z^{2 n+2} d z^{2 n+1} .
$$

Let $\left\{U_{i}\right\}$ be an open covering of $P_{2 n+1}(C)$ and $s_{i}$ a holomorphic crosssection of the principal bundle $C^{2 n+2}-\{0\}$ over $U_{i}$. Set $\omega_{i}=s_{i}^{*}(\omega)$. Then $\left\{\omega_{i}\right\}$ defines a complex contact structure on $P_{2 n+1}(C)$. Considering $C^{2 n+2}$ as the real $(4 n+4)$-dimensional vector space $R^{4 n+4}$, we obtain the $(4 n+3)$-dimensional real projective space $P_{4 n+3}(R) . P_{4 n+3}(R)$ is a principal fibre bundle over $P_{2 n+1}(C)$ with structure group $U(1)$. Every odd dimensional real projective space is a real contact manifold (see for instance [4]). The standard real contact form on $P_{4 n+3}(R)$ is the one derived from the contact form of $P_{2 n+1}(C)$ in the manner described in the proof of (3).

(2) Complex projective co-tangent bundles

Let $V$ be a complex manifold of dimension $n+1$ and $\omega$ a holomorphic 1 -form on the dual complex tangent bundle $\tilde{T}(V)$ (=the space of complex co-tangent vectors) defined by

$$
\omega(u)=v(\delta \pi(u)), \quad u \in T_{v}(\tilde{T}(V))
$$

where $\pi$ is the projection of $\widetilde{T}(V)$ onto $V$ and $\delta \pi$ is the differential of $\pi ; \delta \pi: T(\tilde{T}(V)) \rightarrow T(V)$. In terms of local coordinate $z^{0}, z^{1}, \cdots, z^{n}$ of $V$ and the induced coordinate $z^{0}, z^{1}, \cdots, z^{n}, \zeta_{0}, \zeta_{1}, \cdots, \zeta_{n}$ of $\tilde{T}(V)$,

$$
\omega=\zeta_{0} d z^{0}+\zeta_{1} d z^{1}+\cdots+\zeta_{n} d z^{n} .
$$

The fibre of $\tilde{T}(V)$ being the $(n+1)$-dimensional complex vector space, we construct in a natural way a fibre bundle over $V$ whose 
fibre is the $n$-dimensional complex projective space (complex projective co-tangent bundle). This bundle of complex dimension $2 n+1$ is our $M$. Considering the fibre of $T(V)$ as the $(2 n+2)$-dimensional real vector space, we take as $P$ the co-tangent sphere bundle over $V$ (i.e., the fibre of $P$ is a sphere in the fibre of $\widetilde{T}(V)$ ).

$\tilde{T}(V)-V$ is the principal fibre bundle associated with a line bundle $L$ over $M$. The definition of the complex contact structure on $M$ is similar to the one in the first example. The classical real contact structure on the co-tangent sphere bundle $P$ is the one derived from the complex contact structure on the complex projective co-tangent bundle $M$ as described in the proof of (3).

\title{
BIBLIOGRAPHY
}

1. A. Borel and F. Hirzebruch, Characteristic classes and homogeneous spaces, I, Amer. J. Math. vol. 80 (1958) pp. 458-538.

2. S. S. Chern, Pseudo-groupes continus infinis, Colloque de Geométrie Diff., Strasbourg 1953.

3. C. Chevalley, Theory of Lie groups I, Princeton, 1946.

4. J. W. Gray, Some global properties of contact structures, to appear.

Institute for Advanced Study

\section{FIXED POINTS FOR MULTI-VALUED FUNCTIONS ON SNAKE-LIKE CONTINUA}

\author{
RONALD H. ROSEN ${ }^{1}$
}

1. Introduction. A multi-valued function from a space $X$ into a space $Y$ is a point to set correspondence. Hamilton has shown that snake-like continua have the fixed point property with respect to maps [2]. Ward has extended this to show that snake-like continua have the fixed point property with respect to continuous multivalued functions [6]. The results of this paper establish that a more general class of spaces, those which are inverse limits of arcs, have even stronger properties with respect to multi-valued functions. ${ }^{2}$

2. Multi-valued functions. All functions are multi-valued unless otherwise indicated. A map will always be a continuous single-valued function.

Presented to the Society, April 19, 1958; received by the editors June 5, 1958.

1 National Science Foundation Fellow.

2 The author wishes to thank Professor E. R. Fadell for his encouragement and for reading and criticizing this paper. 